\title{
Granulocyte-colony stimulating factor- associated aortitis in a woman with advanced breast cancer: a case report and review of the literature
}

\author{
Hideko Hoshina ${ }^{1,2^{*}}$ (1) and Hiroyuki Takei ${ }^{1}$
}

\begin{abstract}
Background: Granulocyte-colony stimulating factor (G-CSF) is increasingly been used to prevent febrile neutropenia (FN) associated with the administration of chemotherapy for various cancers. The most common adverse effects of G-CSF are bone pain and injection-site reactions and aortitis has rarely been reported. We report herein a rare case of G-CSF associated with aortitis in a woman with advanced breast cancer.

Case presentation: A 72-year-old woman with estrogen receptor-negative human epidermal growth factor 2-positive breast cancer with distant metastases in the lung was admitted. Her treatment was initiated with docetaxel in combination with trastuzumab and pertuzumab followed by the supportive use of a long-acting G-CSF, pegfilgrastim. After administration of pegfilgrastim on day 5, the patient had an intermittent fever (body temperature up to $39.6^{\circ} \mathrm{C}$ ) on day 9 which continued irrespective of taking levofloxacin. She visited our outpatient clinic on day 13 with no objective symptoms other than fever. Laboratory tests revealed a high neutrophil count $(15,000 / \mu \mathrm{l})$ and a high Creactive protein (CRP) level $(46.35 \mathrm{mg} / \mathrm{dl})$ without any other abnormalities. There was no response upon administration of antimicrobial agents. An 18F-fluorodeoxyglucose-positron emission tomography/computed tomography (FDG-PET/CT) revealed thickening of the wall of the descending thoracic aorta and left pleural effusion. Therefore, thoracic aortitis induced by pegfilgrastim was suspected. On day 19, the fever resolved spontaneously followed by a gradual reduction in the neutrophil count and CRP level. In the follow-up $C T$, the aortic wall thickness and pleural effusion had disappeared.

Conclusions: G-CSF may cause aortitis due to stimulation of the production of inflammatory cytokines. In case of high continuous fever after administration of pegfilgrastim, aortitis should be suspected unless there are other infectious findings.
\end{abstract}

Keywords: Granulocyte-Colony stimulating factor (G-CSF), Aortitis, Breast Cancer, Pegfilgrastim, Filgrastim

\section{Background}

In 2014, a long-acting granulocyte-colony stimulating factor (G-CSF) was approved for breast cancer by the national health insurance in Japan after which it has increasingly been administered to prevent febrile neutropenia (FN) without hospitalization. The most common adverse effects of G-CSF include bone pain and injection-site reactions [1]. G-CSF itself has no negative

\footnotetext{
* Correspondence: s8083@nms.ac.jp

'Department of Breast Surgery and Oncology, Nippon Medical School, 1-1-5 Sendagi, Tokyo, Bunkyo 113-8603, Japan

${ }^{2}$ Department of Breast Surgery, Kikuna Memorial Hospital, Kikuna 4-4-27

Kouhoku, Yokohama, Kanagawa 222-0011, Japan
}

effects on cancer treatment. Furthermore, it has a favorable effect on maintaining a high relative dose intensity to cure the disease. On the other hand, according to the Japanese Adverse Drug Event Report (JADER) provided by the Pharmaceuticals and Medical Devices Agency (PMDA), aortitis is considered as one of the adverse effects of G-CSF although it has rarely been reported. Here, we report a case of aortitis induced by long-acting G-CSF administration to prevent FN in a woman with advanced breast cancer.

(c) The Author(s). 2019 Open Access This article is distributed under the terms of the Creative Commons Attribution 4.0 International License (http://creativecommons.org/licenses/by/4.0/), which permits unrestricted use, distribution, and reproduction in any medium, provided you give appropriate credit to the original author(s) and the source, provide a link to the Creative Commons license, and indicate if changes were made. The Creative Commons Public Domain Dedication waiver (http://creativecommons.org/publicdomain/zero/1.0/) applies to the data made available in this article, unless otherwise stated. 


\section{Case presentation}

A 72-year-old-woman with breast cancer who had already initiated treatment with chemotherapy (first cycle) visited our outpatient clinic with a chief complaint of high fever. She had no previous illness and no particular family history. On clinical examination, she was diagnosed with a clinical stage IV (T4d N2a M1) right breast cancer. A core needle biopsy revealed estrogen receptor-negative and human epidermal receptor 2positive invasive ductal carcinoma of the right breast accompanied with lymph nodes metastases in the ipsilateral axilla. Computed tomography revealed distant metastases in the lungs (Fig. 1).

A chemotherapy regimen consisting of docetaxel $75 \mathrm{mg} / \mathrm{m}^{2}$, trastuzumab $8 \mathrm{mg} / \mathrm{m}^{2}$, and pertuzumab $840 \mathrm{mg}$ was administered with dexamethasone $16.5 \mathrm{mg}$ on day 1 . Dexamethasone $16 \mathrm{mg}$ was orally administered on days 2 to 4 . As per the current guidelines, G-CSF administration is not recommended with the docetaxel regimen. However, G-CSF administration was chosen to ensure safer management of the elderly female patient with advanced-stage breast cancer. Therefore, pegfilgrastim, a long-acting G-CSF was subcutaneously administered on day 5. The patient complained of a high fever (body temperature up to $39.6{ }^{\circ} \mathrm{C}$ ) in the morning on day 9 (day 5 of pegfilgrastim administration). Since then, the intermittent high fever persisted in the morning despite administration of levofloxacin which was prescribed for FN. The patient came to our outpatient clinic on day 13 (day 9 of pegfilgrastim administration) with high fever without any other subjective symptoms.

The patient was conscious, physically well, and showed no infectious manifestations. Laboratory tests revealed a high neutrophil count $(15,000 / \mu \mathrm{l})$ and a high C-reactive protein (CRP) level $(46.35 \mathrm{mg} / \mathrm{dl})$ without any other abnormalities. Influenza antigen test was negative, and urinalysis was clear. Anti-nuclear antibody (ANA), myeloperoxidase-anti-neutrophil cytoplasmic antibody (MPO-ANCA), and serine proteinase3-anti-neutrophil cytoplasmic antibody (PR3-ANCA) were found to be negative later. However, interleukin-6 was slightly elevated $(25.6 \mathrm{pg} / \mathrm{ml})$. She continued to receive antibiotics (cefcapene pivoxil hydrochloride hydrate) because of suspected suffering an infectious disease although blood culture was negative. On day 15, 18F-fluorodeoxyglucosepositron emission tomography/computed tomography (FDG-PET/CT) was initially planned for identification of distant metastasis. However, chemotherapy was undertaken before FDG-PET/CT because a delay of initiation of chemotherapy was deemed inappropriate. FDG-PET/CT was performed at an initially reserved date in order to evaluate the presence of distant metastases except lung metastases. It revealed thickened wall of the descending thoracic aorta with an abnormal FDG uptake accompanied by
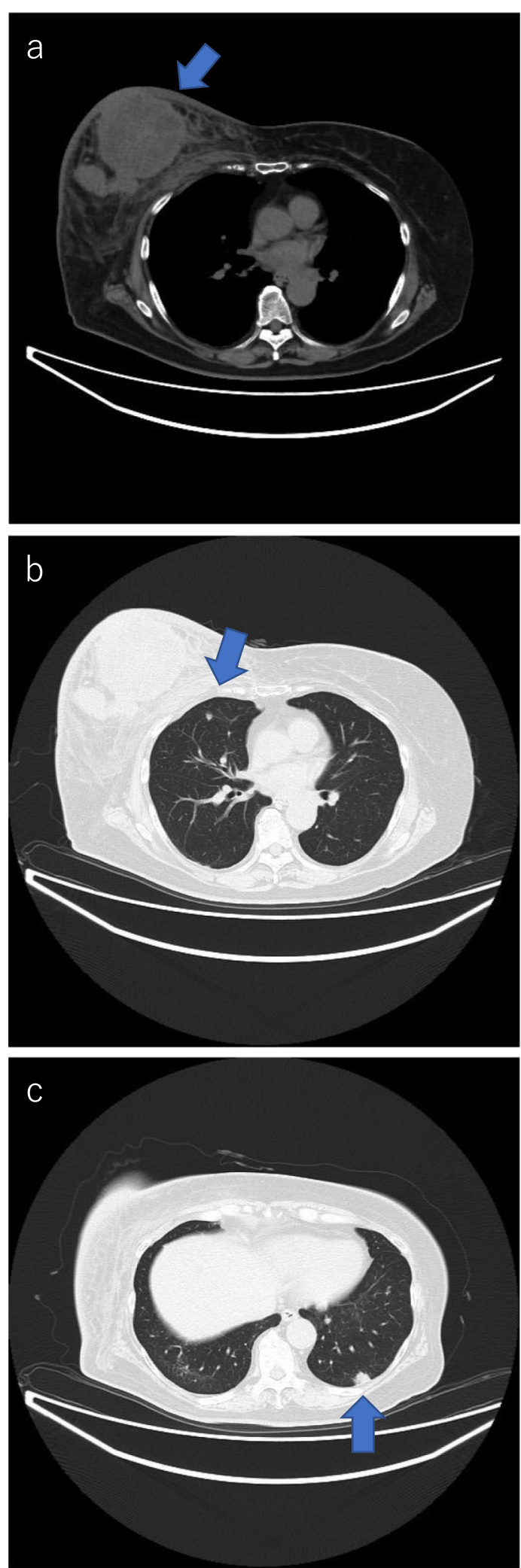

Fig. 1 CT before chemotherapy shows left breast tumor, normal aorta (a), and multiple lung metastases $(\mathbf{b}, \mathbf{c})$ 
left pleural effusion (Fig. 2). However, lung metastases disappeared, and we diagnosed the case as G-CSF-associated aortitis by the FDG-PET/CT.

On day 19, the fever reduced spontaneously. On day 21, the neutrophil count and CRP level reduced to 4940/ $\mu \mathrm{l}$ and $13.29 \mathrm{mg} / \mathrm{dl}$, respectively. The second cycle of chemotherapy was initiated with $30 \%$ reduced dose of docetaxel administered to the patient without pegfilgrastim. In the absence of pegfilgrastim administration, the docetaxel was reduced to $30 \%$ to ensure a safer management. The follow-up CT revealed the disappearance of both aortic wall thickness and pleural effusion. On day 1 of the third cycle of chemotherapy, the neutrophil count and CRP level were almost within the normal limits (4900/ $\mu \mathrm{l}$ and $1.87 \mathrm{mg} / \mathrm{dl}$, respectively). She has been continuing chemotherapy without any further complain of fever.

\section{Discussion and conclusion}

In Japan, G-CSF-associated aortitis is very rare and occurs in just $0.47 \%$ of all cases of G-CSF administration based on the data from JADER [2]. In patients with cancer, aortitis occurs more frequently during chemotherapy with concomitant G-CSF compared to chemotherapy without G-CSF. The incidence of aortitis does not correlate with the type or regimen of chemotherapy. It has been reported more frequently in males than in females. In the United States of America, G-CSFassociated aortitis has been confirmed only in 15 cases as reported by the Adverse Event Reporting System (AERS) of the Food and Drug Administration (FDA) [3]. In these 15 cases, there was no correlation with the type of chemotherapy or gender. Except for five patients, all others recovered spontaneously.

G-CSF facilitates the differentiation and growth of neutrophils. However, it also stimulates the production of inflammatory cytokines [4] which may cause arteriosclerosis [5], aneurysm [6], and arteritis [7, 8]. Aortitis is classified into non-infectious and infectious and most of the non-infectious aortitis is caused by autoimmune disease relative to inflammatory cytokines [9]. In the present case, we excluded autoimmune disease because all of ANA, MPO-ANCA, and PR3-ANCA were within normal levels. We searched previously reported articles including abstracts by using the keywords "G-CSF" and "aortitis" in PubMed and CiNii (Citation Information by National Institute of Informatics). We also checked the references cited in the original articles, and finally identified 10 cases of G-CSF-associated aortitis including the present case (Table 1). The primary diseases included four breast cancer cases [10,11], two lung cancer cases $[12,13]$, and one case of ovarian cancer [14]. G-CSF was used to prevent chemotherapy-induced $\mathrm{FN}$ in these cases. Additionally, there were two bone marrow donors
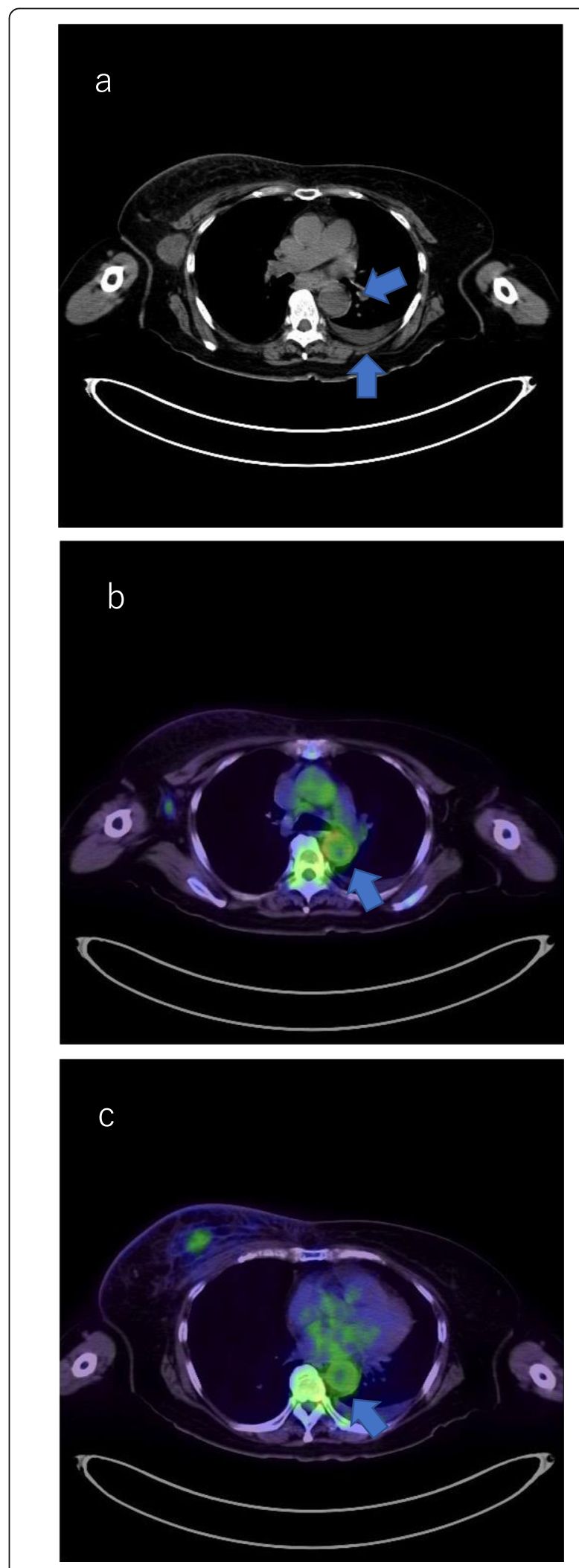

Fig. 2 FDG-PET/CT shows the thickened wall of the thoracic aorta and left pleural effusion (a) with abnormal uptake of FDG (b, c) 


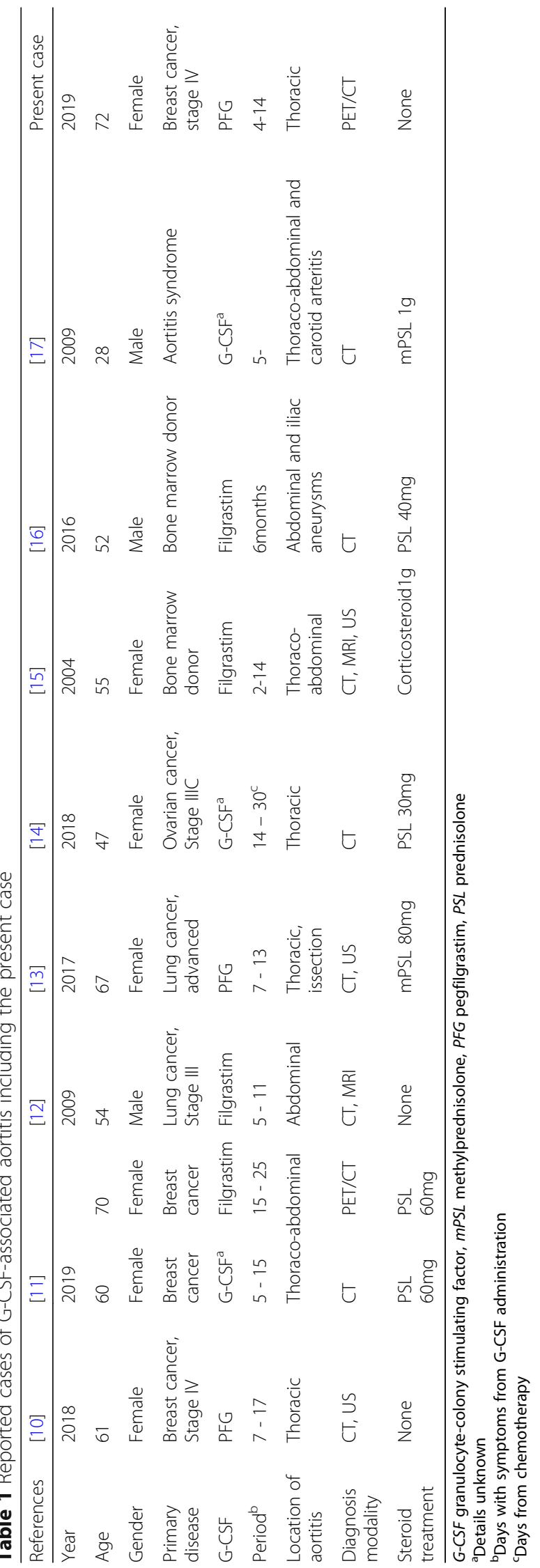


$[15,16]$, and one case of drug-induced agranulocytosis. The latter case was induced by trimethoprim/sulfamethoxazole which was administered for aortitis syndrome [17].

All cases were reported after 2004 suggesting that this disease is recently been recognized. All cases showed good performance status even with high fever and high CRP levels. In all the cases, aortitis was diagnosed by CT scan, FDG-PET/CT, magnetic resonance imaging (MRI), or ultrasound. In seven cases including the present case, high fever was noticed within 7 days of G-CSF administration. There were two cases of different arterial diseases other than aortitis (one case of iliac artery aneurysm and one case of dissection of descending aorta). It is unclear whether these arterial disorders correlated with G-CSF administration. Seven cases were treated with steroids $(30-80 \mathrm{mg} /$ day of oral prednisolone or $1 \mathrm{~g} /$ day of methylprednisolone). However, the high fever persisted for 7-17 days despite the use of steroids. On the other hand, the high fever persisted for 7-11 days without administration of steroids. There was no difference in the time to remission of aortitis with or without the use of steroids.

Interestingly, the five cancer cases where G-CSF was administered to prevent FN were advanced cancers. This signifies that inflammatory cytokines might be produced in larger quantities in advanced-stage cancer than in early-stage cancer. Accordingly, aortitis in patients with advanced-stage cancer should be considered as one of the differential diagnoses if there are long-lasting high fever and high CRP level after administration of G-CSF to prevent $\mathrm{FN}$ unless there are significant infectious manifestations.

\section{Abbreviations}

AERS: Adverse Event Reporting System; ANA: Anti-nuclear antibody; CiNii: Citation Information by National Institute of Informatics; CRP: C-reactive protein; FDA: Food and Drug Administration; FDG-PET/CT: 18Ffluorodeoxyglucose-positron emission tomography/computed tomography; FN: Febrile neutropenia; G-CSF: Granulocyte-colony stimulating factor; JADER: The Japanese Adverse Drug Event Report; MPO-ANCA: Myeloperoxidase-antineutrophil cytoplasmic antibody; MRl: Magnetic resonance imaging; PR3ANCA: Serine proteinase3-anti-neutrophil cytoplasmic antibody

\section{Acknowledgements}

We would like to thank Editage (www.editage.jp) for English language editing.

\section{Authors' contributions}

$\mathrm{HH}$ designed and drafted the work and HT revised it. Both authors approved the final manuscript.

\section{Funding}

Not applicable

\section{Availability of data and materials}

Not applicable

Ethics approval and consent to participate

Not applicable

\section{Consent for publication}

H.H informed the patient that clinical and personal details of the patient would be published on a journal, and a written informed consent was obtained.

\section{Competing interests}

The authors declare that they have no competing interests.

Received: 12 July 2019 Accepted: 26 November 2019

Published online: 16 December 2019

\section{References}

1. Renner P, Milazzo S, Liu JP, Zwahlen M, Birkmann J, Horneber M. Primary prophylactic colony-stimulating factors for the prevention of chemotherapyinduced febrile neutropenia in breast cancer patients. Cochrane Database Syst Rev. 2012;10:CD007913.

2. Oshima Y, Takahashi S, Tani K, Tojo A. Granulocyte colony-stimulating factor-associated aortitis in the Japanese adverse drug event report database. Cytokine. 2019;119:47-51.

3. Lardieri A, McCulley L, Christopher Jones S, Woronow D. Granulocyte colony-stimulating factors and aortitis: a rare adverse event. Am J Hematol. 2018;93(10):E333-6.

4. Pajkrt D, Manten A, van der Poll T, Tiel-van Buul MM, Jansen J. Wouter ten Cate J, et al. modulation of cytokine release and neutrophil function by granulocyte colony-stimulating factor during endotoxemia in humans. Blood. 1997:90(4):1415-24.

5. Haghighat A, Weiss D, Whalin MK, Cowan DP, Taylor WR. Granulocyte colony-stimulating factor and granulocyte macrophage colony-stimulating factor exacerbate arterosclerosis in apolipoprotein E-deficient mice. Circulation. 2007:115(15):2049-54.

6. Ye P, Chen W, Wu J, Huang X, Li J, Wang S, et al. GM-CSF contributes to aortic aneurysms resulting from SMAD3 deficiency. J Clin Invest. 2013;123(5): 2317-31.

7. Umeda M, Ikenaga J, Koga T, Koga T, Michitsuji T, Shimizu T, et al. Giant cell arteritis which developed after the administration of granulocyte-colony stimulating factor for cyclic neutropenia. Intern Med. 2016;55:2291-4.

8. Fukui $\mathrm{S}$, Kuwahara-Takaki A, Ono N, Sato S, Koga T, Kawashiri SY, et al. Serum levels of fibroblast growth factor-2 distinguish Takayasu arteritis from giant cell arteritis independent of age at diagnosis. Sci Rep. 2019;9(1):688.

9. Bossone E, Pluchinotta FR, Andreas M, Blanc P, Citro R, Limongelli G, et al. Aortitis. Vasc Pharmacol. 2016;80:1-10.

10. Chino T, Oba T, Yamamoto K, Takekoshi D, lesato A, Ito T, et al. A Case of arteritis that developed after pegfilgrastim administration during chemotherapy for breast cancer. Jpn J Cancer Chemother. 2018;45(12): 1771-4 (Japanese).

11. Parodis I, Dani L, Notarnicola A, Martenhed G, Fernström P, Matikas A, et al. G-CSF-induced aortitis: two cases and review of the literature. Autoimmun Rev. 2019:18(6):615-20.

12. Adiga GU, Elkadi D, Malik SK, Fitzpatrick JD, Madajewicz S. Abdominal Aortitis after use of granulocyte Colony-stimulating factor. Clin Drug Investig. 2009;29(12):821-5.

13. Sato Y, Kaji S, Ueda H, Tomii K. Thoracic aortitis and aortic dissection following pegfilgrastim administration. Eur J Cardiothorac Surg. 2017;52(5): 993-4.

14. Hiranuma K, Kusunoki S, Fujino K, Hirayama T, Ota T, Terao Y. Drug-induced aortitis in a patient with ovarian cancer treated with bevacizumab combination therapy. Taiwan J Obstet Gynecol. 2018;57(5):750-2.

15. Darie $C$, Boutalba $S$, Fichter $P$, Huret JF, Jaillot $P$, Gerenton $S$, et al. Aortitis after G-CSF injections. Rev Med Interne. 2004;25(3):225-9 (French).

16. Miller EB, Grosu R, Landau Z. Isolated abdominal aortitis following administration of granulocyte colony stimulating factor (G-CSF). Clin Rheumatol. 2016;35(6):1655-7.

17. Suzuki $E$, Iwadate $H$, Saito $R$, Kobayashi $H$, Watanabe $H$, Ohira $H$. A case of Aortitis syndrome that recurred after G-CSF administration (Japanese). Jpn J Clin Immunol. 2009;37:159.

\section{Publisher's Note}

Springer Nature remains neutral with regard to jurisdictional claims in published maps and institutional affiliations. 